\title{
Laparoscopically-Assisted Repair of a Small Bowel Perforation Secondary to Multiple Metastases of Undifferentiated Pleomorphic Sarcoma
}

\author{
Masaki Morimoto, Soichiro Honjo, Teruhisa Sakamoto, Takuki Yagyu, Ei Uchinaka, Takehiko Hanaki, \\ Joji Watanabe, Tomoyuki Matsunaga, Manabu Yamamoto, Yoji Fukumoto, Naruo Tokuyasu and \\ Yoshiyuki Fujiwara \\ Division of Surgical Oncology, Department of Surgery, School of Medicine, Tottori University Faculty of Medicine, Yonago 683-8504, \\ Japan
}

\begin{abstract}
We present a very rare case of a laparoscopicallyassisted repair of a small bowel perforation secondary to multiple metastases of undifferentiated pleomorphic sarcoma from the posterior mediastinum. A 46-yearold man presented with middle to upper abdominal pain during chemotherapy for lung metastases from undifferentiated pleomorphic sarcoma. Computed tomography revealed intra-abdominal free air, and emergency laparoscopy was performed. Consequently, a perforation was detected in the jejunum, and partial jejunal resection was performed by mini-laparotomy. Pathological examination demonstrated an ulcerated tumor with perforation and four additional tumors in the resected jejunum. Pathological examination also revealed undifferentiated pleomorphic sarcoma in all five tumors. To our knowledge, our case is the first report of a laparoscopically-assisted repair of a small bowel perforation secondary to metastasis of undifferentiated pleomorphic sarcoma. Although the perforation site was unclear preoperatively, laparoscopic observation readily identified the lesion in this patient. Therefore, minimally invasive surgery could be performed successfully with mini-laparotomy following laparoscopic observation. Laparoscopic techniques for the small intestine are viable options, even in acute and uncommon situations, and small bowel perforation secondary to metastasis should be considered in patients with undifferentiated pleomorphic sarcoma and acute abdomen.
\end{abstract}

Key words laparoscopy; malignant fibrous histiocytoma; sarcoma; small intestine

Undifferentiated pleomorphic sarcoma (UPS) is a relatively common neoplasm that most frequently affects the

Corresponding author: Masaki Morimoto, MD

2s.morimoto@gmail.com

Received 2020 February 13

Accepted 2020 March 5

Online published 2020 March 18

Abbreviations: CT, computed tomography; MFH, malignant fibrous histiocytoma; UPS, undifferentiated pleomorphic sarcoma soft tissues. Soft tissue sarcoma usually metastasizes to the lungs; tumors arising in the abdominal cavity more commonly metastasize to the liver and peritoneum. We describe an extremely rare case of small intestinal perforation secondary to metastatic UPS.

\section{PATIENT REPORT}

A 46-year-old man presented with middle to upper abdominal pain while receiving chemotherapy for lung metastases from posterior mediastinal UPS. He had no significant medical history except for the UPS. He also had no history of previous abdominal surgery. The UPS was resected and diagnosed 2 years earlier, and the right lung recurrence occurred within 2 months after the resection. Therefore, he underwent partial resection of his right upper lobe for the metastatic lesion. Unfortunately, he was diagnosed with newly-arising multiple metastases in both lungs soon after the second resection, and chemotherapy with doxorubicin $\left(60 \mathrm{mg} / \mathrm{m}^{2}\right)$ was the first-line approach. After 4 months, pazopanib $(800 \mathrm{mg} /$ day for 2 days) was started as the second-line approach nine days before the initial consultation to us, because of disease progression. On initial consultation, the patient's body temperature was $37.3^{\circ} \mathrm{C}$, his heart rate was 92 beats/min, blood pressure was $129 / 78 \mathrm{mmHg}$, and $\mathrm{SpO}_{2}$ was $97 \%$ breathing room air. No tumor was detected in the abdomen, but there was tenderness in the epigastric and periumbilical regions with peritoneal signs. His bowel sounds were normal. Laboratory examination revealed that the patient's C-reactive protein level was $9.38 \mathrm{mg} / \mathrm{dL}$ and his white blood cell count was 11,300 cells $/ \mu \mathrm{L}$. Plain and contrast-enhanced abdominal computed tomography (CT) revealed intraabdominal free air under the diaphragm, in the hepatic portal region, and around the right paracolic sulcus (Fig. 1). It was impossible to identify the site of perforation; however, the wall of the duodenum was slightly unclear and was a suspected perforation (Fig. 2).

To diagnose and repair the gastrointestinal perforation, we performed emergency laparoscopy under general anesthesia. During the surgery, a 12-mm trocar was inserted through an umbilical incision using an open 


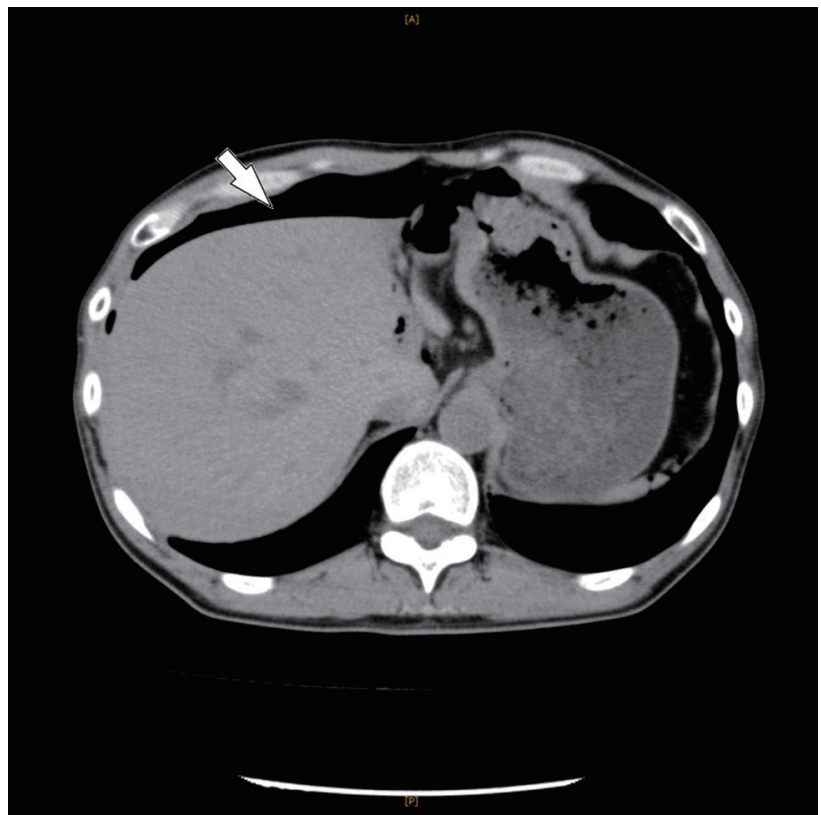

Fig. 1. Preoperative CT. White arrow indicates free air under the diaphragm.

technique. A flexible 10-mm laparoscope was inserted into the abdomen via the trocar, and pneumoperitoneum was established. Subsequently, 5-mm and $10-\mathrm{mm}$ trocars were placed in the left and right supraumbilical region, respectively.

There was no evidence of perforation or pus around the stomach, duodenum, or intestinal sections in the pelvic cavity. However, an approximately $10-\mathrm{mm}$ perforation was detected in the jejunum $20 \mathrm{~cm}$ distal to the ligament of Treitz (Fig. 3). No tumors were revealed by laparoscopic observation. The umbilical port incision was extended for $4 \mathrm{~cm}$, and a mini-laparotomy was performed. Two more tumors around the perforation were identified, and partial jejunal resection, including all three lesions, with a functional end-to-end anastomosis was performed. The operative time was $94 \mathrm{~min}$, and the amount of intraoperative bleeding was $30 \mathrm{~g}$. The patient was discharged on postoperative day 5 without complications.

Pathological examination demonstrated an ulcerated tumor with perforation and another four tumors in the resected jejunum (Fig. 4). Pathological examination also revealed UPS with bizarre cells in all five tumors (Fig. 5).

Postoperatively, the patient experienced no recurrence of perforation, but he died 2 months after the operation as a result of cancer progression. Autopsy confirmed multiple metastases of UPS (lung, pleura, pericardium, liver, pancreas, peritoneum, mesentery, and paraaortic lymph node).

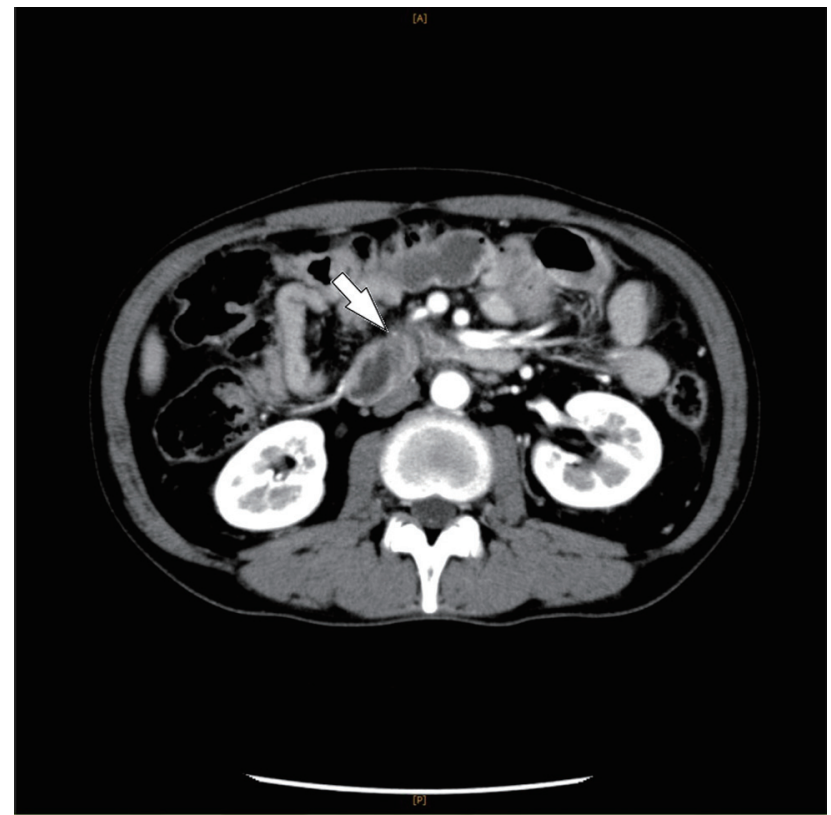

Fig. 2. Preoperative CT. White arrow indicates that the wall of the duodenum was slightly unclear, indicating a possible perforation.

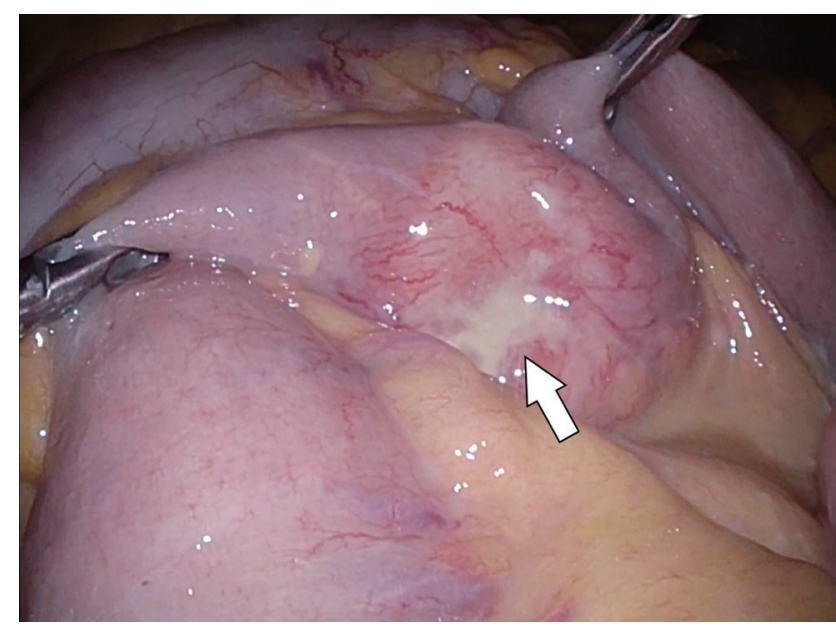

Fig. 3. The perforation site covered with pus was detected in the jejunum laparoscopically (white arrow).

\section{DISCUSSION}

Common subtypes of soft tissue sarcomas include UPS, gastrointestinal stromal tumor, liposarcoma, and leiomyosarcoma. ${ }^{1}$ Extremities (43\%), the trunk $(10 \%)$, viscera $(19 \%)$, retroperitoneum $(15 \%)$, and head and neck $(9 \%)$ are the most common primary sites. ${ }^{2}$ Generally, surgical resection with appropriately negative margins is the standard primary treatment for most patients with soft tissue sarcomas, according to the National Comprehensive Cancer Network guidelines. ${ }^{3}$ For unresectable or metastatic disease, chemotherapy 


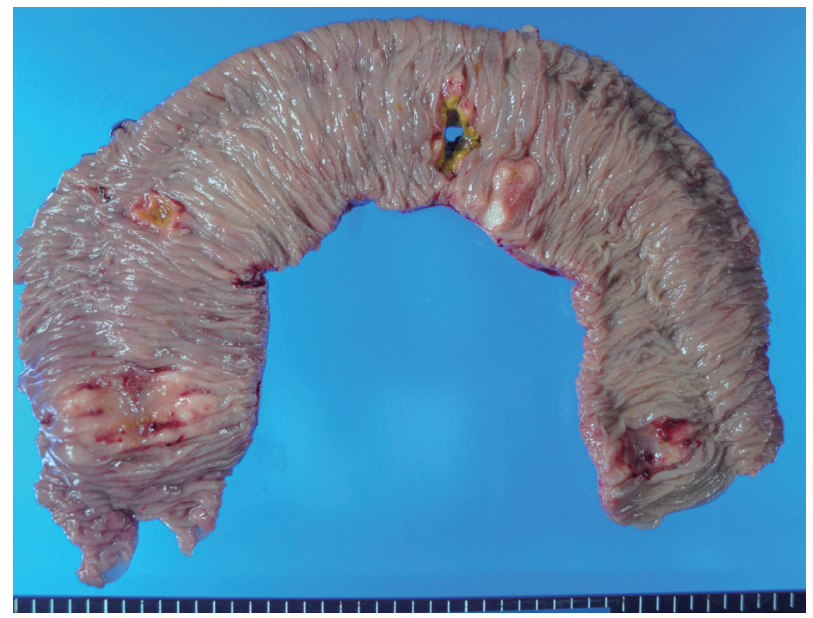

Fig. 4. The resected jejunum including an ulcerated tumor with perforation and four additional tumors.

with single agents (dacarbazine, doxorubicine, epirubicin, or ifosfamide) or anthracycline-based combination regimens have been widely used. More recently, a number of targeted therapies have shown promising results in patients with certain types of advanced or metastatic soft tissue sarcoma. ${ }^{3}$

In 2013, the World Health Organization classification of soft tissue tumors was updated. With the advent of advanced diagnostics, the term "malignant fibrous histiocytoma (MFH)" became somewhat antiquated as well as imprecise and was superseded by the term "undifferentiated pleomorphic sarcoma" in this revision. ${ }^{4}$ These tumors have no specific markers for diagnosis and have in common marked cytological and nuclear pleomorphism, often with bizarre tumor giant cells, admixed with spindle cells and often rounded histiocytelike cells (which may have foamy cytoplasm) in varying proportions. 5,6

MFH occurs principally as a mass on an extremity (lower extremity $49 \%$, upper extremity $19 \%$ ) or in the abdominal cavity or retroperitoneum $(16 \%)$ of adults (peak incidence, $61-70$ years of age). ${ }^{7}$ The reported local recurrence rate of the tumor is $44 \%$, and the metastatic rate is $42 \%$; furthermore, metastasis is most frequently to the lung $(82 \%)$ and lymph nodes $(32 \%)^{7}$

In this report, we presented a case of a laparoscopically-assisted repair of a small bowel perforation secondary to multiple metastases of UPS from the posterior mediastinum. Historically, laparotomy has been the intervention of choice for acute abdomen. However, laparoscopic exploration has emerged as a viable option to identify and treat the source of perforation because of its minimal invasiveness. In particular, perforated peptic ulcer, ${ }^{8}$ acute cholecystitis ${ }^{9}$ and acute appendicitis ${ }^{10}$ are treated laparoscopically. In small bowel emergencies, laparoscopy has been adopted as a first-line option in many elective indications of acute small bowel obstruction, and it is also emerging as a feasible alternative to treat this condition, ${ }^{11}$ even though open surgery has been the gold standard therapy for adhesive small bowel obstruction for decades. The best approach for small bowel perforation has been evaluated previously, ${ }^{12}$ and Sinha et al. described 25 patients with small bowel perforation in whom laparoscopic intracorporeal bowel repair was performed and concluded that laparoscopic intervention was safely and efficaciously extended to such patients, especially regarding the lower rate of wound complications. ${ }^{13}$ Additionally, some case reports described the feasibility and advantages of laparoscopy for small bowel perforation secondary to an ingested foreign body. ${ }^{14,15}$ These reports suggest that laparoscopic procedures are also beneficial in the treatment of small bowel perforation. Even though, poor laparoscopic view due to such conditions as severe intestinal distension or adhesion can be a limitation of laparoscopy. Conventional laparotomy should be considered for the safety of operation in cases with poor laparoscopic view.

Gastrointestinal perforation is a critical adverse event with molecular-targeted therapies such as bevacizumab $^{16}$; however, pazopanib, a small-molecule inhibitor of multiple protein tyrosine kinases that was used in our patient, does not appear to lead to gastrointestinal perforation. ${ }^{17}$

Although malignant UPS/MFH is one of the most common soft tissue sarcomas, metastasis to the small bowel has been reported rarely. To the best of our knowledge, there are 12 reported cases to date, including ours, describing small bowel metastasis of UPS/ MFH (Table 1). The age range in the published studies was $25-73$ years, and 10 patients $(83 \%)$ were men. The patients had various primary sites of malignancy, namely, the extremities, trunk, and viscera. Symptoms related to gastrointestinal bleeding were seen in four patients (44\%), and intussusception was seen in three patients (25\%). All procedures were performed by open laparotomy for small bowel metastasis.

Our literature review also revealed that intestinal perforation secondary to metastasis of UPS/MFH is extremely rare, with only one reported patient. ${ }^{18}$ We believe that our case is the first report of laparoscopicallyassisted repair of a small bowel perforation secondary to UPS metastasis. Although the perforation site was unclear preoperatively, laparoscopy readily identified the lesion in our patient. Therefore, minimally invasive surgery can be performed successfully with minilaparotomy following laparoscopic observation. 
Table 1. Cases of small bowel metastasis in UPS/MFH

\begin{tabular}{|c|c|c|c|c|c|c|c|}
\hline No. & Author & Year & Age (years) & Sex & Primary site & Symptoms & Treatment for metastasis \\
\hline 1 & Wasserman, T. H. & 1974 & 69 & Male & Shoulder & Unknown & None \\
\hline 2 & Wasserman, T. H. & 1974 & 78 & Male & Unknown & Unknown & None \\
\hline 3 & Jun MISONOU & 1990 & 25 & Male & Mediastinum & Tarry stool & Resection (OL) \\
\hline 4 & Gorman, R. C. & 1993 & 47 & Female & Thigh & Intussusception & Resection (OL) \\
\hline 5 & Blaauwwiekel, E. E. & 1997 & 75 & Male & Right foot & Bleeding & Resection (OL) \\
\hline 6 & Kanoh, T. & 1998 & 45 & Male & Right back & Intussusception & Resection (OL) \\
\hline 7 & Tranter, S. E. & 2000 & 73 & Female & Left arm & Intussusception & Resection (OL) \\
\hline 8 & Tamura, M. & 2002 & 73 & Male & Rib & Bleeding & Resection (OL) \\
\hline 9 & Agaimy, A. & 2007 & 30 & Male & Left atrium & $\begin{array}{l}\text { Abdominal pain, } \\
\text { Bleeding }\end{array}$ & Resection (OL) \\
\hline 10 & Oguri, Y. & 2018 & 70 & Male & Stomach & Abdominal fullness & Resection (OL) \\
\hline 11 & Plestina, S. & 2019 & 57 & Male & Lung & Perforation & Resection (OL) \\
\hline 12 & Our case & 2019 & 46 & Male & Mediastinum & Perforation & Resection (laparoscopic) \\
\hline
\end{tabular}

MFH, malignant fibrous histiocytoma; OL, open laparotomy; UPS, undifferentiated pleomorphic sarcoma.

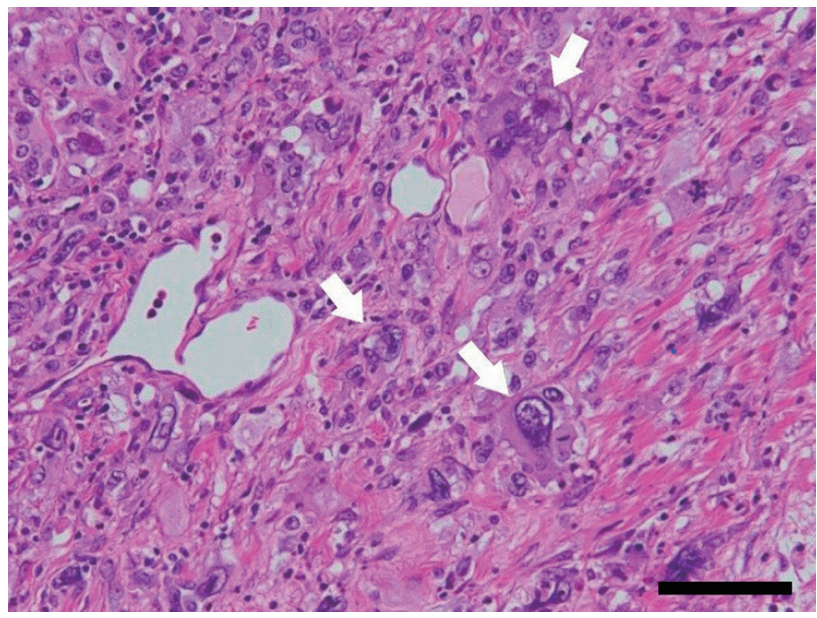

Fig. 5. Pathological examination showing UPS with bizarre cells (white arrows) in all five tumors. Bar $=100 \mu \mathrm{m}$.

Generally, laparoscopic procedures for acute abdomen are limited to severe states related to a primary disease and/or the surgeon's experience, and conversion to open laparotomy is sometimes needed. ${ }^{19,20}$ However, studies have reported the potential benefits of laparoscopic surgery regarding reduced morbidity, less requirement for analgesics, shorter hospital stay, and quicker recovery compared with open surgery. ${ }^{13}$ As an example, fortunately, our patient had a good outcome following the laparoscopically-assisted procedure, even though his was an extremely rare case of small bowel perforation secondary to UPS metastasis. Laparoscopic techniques for the small intestine are viable options even in acute and uncommon situations, and small bowel perforation secondary to UPS metastasis should be considered in patients with UPS and acute abdomen.

Acknowledgments: The authors thank the nurses, doctors, and other staff responsible for the patient's care at Tottori University Hospital. We thank Jane Charbonneau, DVM, from Edanz Group (www.edanzediting.com/ac) for editing a draft of this manuscript.

The authors declare no conflict of interest.

\section{REFERENCES}

1 What Is a Soft Tissue Sarcoma? [Internet] Atlanta: American Cancer Society; c2020 [cited 2020 Jan 25]; Available from: https://www.cancer.org/cancer/soft-tissue-sarcoma/about/softtissue-sarcoma.html

2 Pazdur RWL, Camphausen KA, Hoskins WJ. Cancer Management:A Multidisciplinary Approach Medical, Surgical, \& Radiation Oncology. 13th ed. Norwalk, CT: UBM Medica LLC; 2011.

3 von Mehren M, Randall RL, Benjamin RS, Boles S, Bui MM, Ganjoo KN, et al. Soft Tissue Sarcoma, Version 2.2018, NCCN Clinical Practice Guidelines in Oncology. J Natl Compr Canc Netw. 2018;16:536-63. DOI: 10.6004/ jnccn.2018.0025, PMID: 29752328

4 Jo VY, Fletcher CDM. WHO classification of soft tissue tumours: an update based on the 2013 (4th) edition. Pathology. 2014;46:95-104. DOI: 10.1097/PAT.0000000000000050, PMID: 24378391

5 Fletcher CDM. Pleomorphic malignant fibrous histiocytoma: fact or fiction? A critical reappraisal based on 159 tumors diagnosed as pleomorphic sarcoma. Am J Surg Pathol. 1992;16:213-28. DOI: 10.1097/00000478-199203000-00001, PMID: 1317996 
6 Fletcher CDMBJ, Hogendoorn PCW, Mertens F. World Health Organization Classification of Tumours: Tumours of Soft Tissue and Bone. Lyon, France: IARC Press; 2013.

7 Weiss SW, Enzinger FM. Malignant fibrous histiocytoma. An Analysis of 200 Cases. Cancer. 1978;41:2250-66. DOI: 10.1002/1097-0142(197806)41:6<2250::AIDCNCR2820410626>3.0.CO;2-W, PMID: 207408

8 Byrge N, Barton RG, Enniss TM, Nirula R. Laparoscopic versus open repair of perforated gastroduodenal ulcer: a National Surgical Quality Improvement Program analysis. Am J Surg. 2013;206:957-63. DOI: 10.1016/j.amjsurg.2013.08.014, PMID: 24112676

9 Knab LM, Boller AM, Mahvi DM. Cholecystitis. Surg Clin North Am. 2014;94:455-70. DOI: 10.1016/j.suc.2014.01.005, PMID: 24679431

10 Kouhia ST, Heiskanen JT, Huttunen R, Ahtola HI, Kiviniemi VV, Hakala T. Long-term follow-up of a randomized clinical trial of open versus laparoscopic appendicectomy. Br J Surg. 2010;97:1395-400. DOI: 10.1002/bjs.7114, PMID: 20632312

11 Di Saverio S, Coccolini F, Galati M, Smerieri N, Biffl WL, Ansaloni L, et al. Bologna guidelines for diagnosis and management of adhesive small bowel obstruction (ASBO): 2013 update of the evidence-based guidelines from the world society of emergency surgery ASBO working group. World J Emerg Surg. 2013;8:42. DOI: 10.1186/1749-7922-8-42, PMID: 24112637

12 Talwar S, Laddha BL, Jain S, Prasad P. Choice of incision in surgical management of small bowel perforations in enteric fever. Trop Gastroenterol. 1997;18:78-9. PMID: 9323924

13 Sinha R, Sharma N, Joshi M. Laparoscopic repair of small bowel perforation. JSLS. 2005;9:399-402. PMID: 16381353
14 Ito S, Tsuchitani Y, Hashimoto S, Uemura T, Katsura K, Abe T, et al. Successful treatment with laparoscopy-assisted surgery for ileal perforation due to an ingested foreign body: A report of two cases. Int J Surg Case Rep. 2019;64:165-9. DOI: 10.1016/j.ijscr.2019.10.018, PMID: 31655289

15 Lunsford KE, Sudan R. Small bowel perforation by a clinically unsuspected fish bone: laparoscopic treatment and review of literature. J Gastrointest Surg. 2012;16:218-22. DOI: 10.1007/s11605-011-1610-y, PMID: 21796463

16 Hurwitz H, Fehrenbacher L, Novotny W, Cartwright T, Hainsworth J, Heim W, et al. Bevacizumab plus irinotecan, fluorouracil, and leucovorin for metastatic colorectal cancer. N Engl J Med. 2004;350:2335-42. DOI: 10.1056/ NEJMoa032691, PMID: 15175435

17 van der Graaf WTA, Blay JY, Chawla SP, Kim DW, Bui-Nguyen B, Casali PG, et al.; EORTC Soft Tissue and Bone Sarcoma Group; PALETTE study group. Pazopanib for metastatic soft-tissue sarcoma (PALETTE): a randomised, double-blind, placebo-controlled phase 3 trial. Lancet. 2012;379:1879-86. DOI: 10.1016/S0140-6736(12)60651-5, PMID: 22595799

18 Pleština S, Librenjak N, Marušić A, Batelja Vuletić L, Janevski Z, Jakopović M. An extremely rare primary sarcoma of the lung with peritoneal and small bowel metastases: a case report. World J Surg Oncol. 2019;17:147. DOI: 10.1186/s12957019-1691-8, PMID: 31426804

19 Lin HF, Lai HS, Lai IR. Laparoscopic treatment of perforated appendicitis. World J Gastroenterol. 2014;20:14338-47. DOI: 10.3748/wjg.v20.i39.14338, PMID: 25339821

20 Ball CG, Kortbeek JB, Kirkpatrick AW, Mitchell P. Laparoscopic appendectomy for complicated appendicitis: an evaluation of postoperative factors. Surg Endosc. 2004;18:969-73. DOI: 10.1007/s00464-003-8262-2, PMID: 15095081 